\title{
Comparison of the efficacy of two types of hemodialysis catheters: the VectorFlow vs. Palindrome catheters
}

\author{
Veisi Ali ${ }^{1}$, Sobhiyeh MohammadReza ${ }^{2, *}$
}

${ }^{1}$ Deparment of Surgery, Imam Reza Hospital, Kermanshah University of medical Sciences, Kermanshah, Iran

${ }^{2}$ Department of Vascular and Endovascular Surgery, Imam Reza Hospital, Kermanshah University of medical Sciences, Kermanshah, Iran

\section{Correspondence}

Sobhiyeh MohammadReza, Department of Vascular and Endovascular Surgery, Imam Reza Hospital, Kermanshah University of medical Sciences,

Kermanshah, Iran

Email: mreza.sobhiyeh@yahoo.com

History

- Received: Mar 15, 2019

- Accepted: Jun 11, 2019

- Published: Jun 30, 2019

DOI :

https://doi.org/10.15419/bmrat.v6i6.551

\section{Check for updates}

\section{Copyright}

( ) Biomedpress. This is an openaccess article distributed under the terms of the Creative Commons Attribution 4.0 International license.

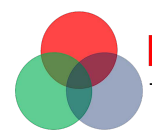

The Open Access Publisher

\begin{abstract}
Introduction: Permanent vascular access via catheters is a critical factor for patients who need long-term hemodialysis. Differences in the design and mechanical factors can affect efficacy and survival of the permanent catheters. Modifications in the designs of the catheters can decrease the likelihood of damaging blood vessels as well as thrombosis formation and malfunction of the catheters. Objective: To compare survival of VectorFlow, complete symmetry of distal tip and offsetting of its side holes, with those of Palindrome, a complete symmetrical catheter. Methods: In this study, 146 CKD (chronic kidney disease) patients who required chronic hemodialysis were divided into VectrFlow (76 subjects) and Palindrome (70 subjects) catheter groups. The patients were followed for six months and the survival rate in each group was determined. Results: Mean ( \pm SD) survival time in the VectorFlow catheter group (6.55 \pm 3.88 months, 2 to 24 months) was longer than in the Palindrome catheter group (5.22 \pm 2.88 months, one to 13 months). After six months, the VectorFlow catheter group (40 cases, 54.8\%) had a higher number of patients, having a functioning catheter in place than the Palindrome group (26 cases, 382\%); $P=0.04$. Conclusion: The VectorFlow hemodialysis catheter had a better survival rate after 6 months when compared to the Palindrome catheter. We recommend using this catheter in CKD patients, who require a long-term permanent catheter.

Key words: End stage renal disease, hemodialysis, permanent catheter, hemodialysis catheter, VectorFlow, Palindrome
\end{abstract}

\section{INTRODUCTION}

In case arterio-venous fistula (AVF) is malfunctioning and has complications, tunneled cuffed hemodialysis catheters are necessary for CKD (chronic kidney disease) patients, who require permanent catheters ${ }^{1}$. Many guidelines recommend that native AVFs are the preferred way for vascular access for hemodialysis patients $^{2}$. However, in several conditions, AVF may not be possible and accessible easily due to anatomical considerations and non-suitable vascular condition. There is an evidence, that the need for hemodialysis permanent catheters, though being the least desirable method for vascular access has been increasing. This mainly happens due to aging of the patient population, which makes vascular preparation difficult ${ }^{3,4}$.

The complications regarding synthetic permanent catheters include thrombosis formation, infection, etc. ${ }^{5}$. An adequate blood flow to deliver proper hemodialysis is critical, when evaluating functioning of catheters; it is suggested that about one-third of cases that require removal or exchange of catheters is owing to a poor blood flow ${ }^{6}$. Optimizing specific mechanical properties of catheters (including the design of the catheter tip), can reduce the afore mentioned complications. Therefore, efforts have been made to design and introduce better catheters.

Permanent catheters, available on the market come in different shapes and types. They differ in their tip design and lumen diameter. These alterations in the design of the catheters are made to prolong survival rate of the catheters ${ }^{7}$. In order to extend the survival rates of permanent catheters, certain strategies such as correcting and designing the catheter tip are implemented.

The Palindrome catheter with symmetrical tip design, was introduced in 2005. It was to overcome shortcomings of previously used catheters with staggered tips ${ }^{8}$. The spiral Z-shaped tip of the Palindrome was designed to decrease blood recycling rate. Previous studies reported efficacy of the Palindrome catheter ${ }^{4,9}$. The VectorFlow catheter for use in hemodialysis patients was cleared by the FDA (Food and Drug Administration) in $2014^{10}$. Although designs of the tips in the VectorFlow and Palindrome catheters are symmetrical, in the VectorFlow, low flow rate in side the holes by helical flowdeflecting interfaces with less shear-induced platelet activation ${ }^{11}$ with separate arterial and venous apertures. The changes in configuration have been made 
to decrease the likelihood of thrombosis formation in this catheter.

The purpose of this study was to compare the survival rate of the Palindrome catheter to that of the VectorFlow catheter for hemodialysis patients. By better understanding long-term outcome of the function of these catheters, the clinicians will use the findings for better clinical decision making.

\section{MATERIALS AND METHODS}

This study was conducted in our tertiary academic medical center in 2017-18. The study population consisted of patients with $\mathrm{CKD}$, who were receiving hemodialysis and were referred to the vascular surgery department for placement of permanent hemodialysis catheter. A total of 185 patients (110 male and 75 female patients) were recruited. Of these, 39 patients were excluded as the patients died (26 cases) or catheter withdrawal. Finally, 146 patients remained for final analyses in the Palindrome group (70 cases) or the VectorFlow group (76 cases). The catheters were inserted into the jugular vein under ultrasound guide.

\section{Data collection}

A checklist was designed to gather the required data, including demographic data, hypertension, and diabetes mellitus; duration of haemodialysis and survival rates of permanent haemodialysis catheters.

\section{Statistical analysis}

Statistics, including frequency (percentage), mean and standard deviation were used to report the descriptive data. To determine efficacy of the catheters the student t-test, or Mann Whitney U test was used. The Chi-square test was used to compare categorical variables between the two groups. Statistical analyses were performed with SPSS software (ver. 16.0) and P values less than $5 \%$ were considered statistically significant.

\section{Ethics}

The patients were informed about the objectives of the study and if agreed, informed consent was obtained. The study protocol was verified by the Research Deputy of Kermanshah University of Medical Sciences, Kermanshah, Iran (IR.KUMS.REC.1379.519).

\section{RESULTS}

A total number of 146 patients in the Palindrome group (70 cases) and the VectorFlow group (76 cases) were included for the final analyses. Table 1 presents comparison of gender, hypertension, and diabetes mellitus. As observed, except for higher proportion of male patients in the Palindrome group, no difference was seen regarding the frequency of hypertension and diabetes mellitus between the two groups.

Mean $( \pm \mathrm{SD})$ survival time in the VectorFlow catheter group ( $6.55 \pm 3.88$ months, 2 to 24 months) was longer than in the Palindrome catheter group (5.22 \pm 2.88 months, one to 13 months).

After six months, the VectorFlow catheter group (40 cases, 54.8\%) had a higher number of patients, having a functioning catheter in place than the Palindrome group (26 cases, $382 \%$ ); $\mathrm{P}=0.04$. Table 2 presents frequency of successful and patent catheters at 3 and 6 months.

\section{DISCUSSION}

In this study, we compared survival rates of two types of permanent hemodialysis catheters with different tip designs in continuous hemodialysis patients. The obtained findings showed that the VectorFlow was superior to the Palindrome. This is attributed to the tip shape of the VectprFlow catheter. Survival rate in the VectorFlow catheter group was higher than that of the Palindrome group.

A previous simulation model study ${ }^{12}$, comparing three brands of permanent catheters with symmetrical tips (VectorFlow, Palindrome, and GalidePath), showed that the VectorFlow catheter had less potential for shear-induced platelet activation. Platelet aggregation, deposition, and activation can cause thrombosis formation. The structure of the tip of the catheter can damage the wall of blood vessels resulting in platelet aggregation and thrombosis formation ${ }^{13}$. In another study ${ }^{10}$, after 4 months of follow-up of two groups of hemodialysis patients who received VectorFlow or Ash Split Cath, it was found that significantly higher percentage of CevtorFlow catheters (about 90\%) were patent when compared to the other group (45\%).

In a study ${ }^{11}$ that followed hemodialysis patients for three months, it was shown that VectorFlow was a safe and effective catheter in these patients with no acute complications. Just three patients out of 46 included subjects developed infection (two cases) and malfunctioning (one patient). Similar to our results that $91.8 \%$ of the patients in VectorFlow group had patent VectorFlow catheter, in the mentioned study ${ }^{11}, 88.8 \%$ of the patients had patent and functioning catheter.

The main advantage of the VectorFlow catheter over others is an improvement of mechanical features, which allows for a decrease of thrombosis formation. 
Table 1: Comparison of gender distribution, hypertension, and diabetes mellitus between two groups of hemodialysis patients, requiring permanent hemodialysis catheter

\begin{tabular}{cllll}
\hline & \multicolumn{2}{c}{ Palindrome catheter $(\mathbf{N}=\mathbf{7 0})$} & VectorFlow catheter $(\mathbf{N}=76)$ & P value \\
Gender & Male & $48(68.6 \%)$ & $39(51.3 \%)$ & 0.03 \\
Female & $22(31.4 \%)$ & $37(48.7 \%)$ & \\
Hypertension & $45(64.3 \%)$ & $59(77.6 \%)$ & 0.07 \\
Diabetes mellitus & $26(37.1 \%)$ & $39(51.3 \%)$ & 0.08 \\
\hline
\end{tabular}

Table 2: Comparison of successful catheters at 3 and 6 months in the two studied groups

\begin{tabular}{llll}
\hline & Palindrome catheter $(\mathbf{N}=70)$ & VectorFlow catheter $(\mathbf{N}=76)$ & P value \\
3-month patency rate & $60(88.2 \%)$ & $67(91.8 \%)$ & 0.48 \\
6-month patency rate & $26(38.2 \%)$ & $40(54.8 \%)$ & 0.04 \\
\hline
\end{tabular}

When blood flow is compromised, causing irregular flow, this phenomenon damages vascular wall and subsequently thrombus is formed ${ }^{7}$. The Palindrome catheter with its spiral end tip has also been shown to have reasonably good functioning. For example, in a study ${ }^{4}$ that included 49 patients with the Palindrome catheter, maximal blood flow was $351 \mathrm{~mL} / \mathrm{min}$ which was higher than in the Permcath group. In addition, only 4 cases $(8.2 \%)$ had dysfunction of the Palindrome catheter. However, in our study, the dysfunction rate was higher and after 3 months only $88.2 \%$ had patent catheters. In another report, six catheters out of 60 catheters $(10 \%)$ required removal or exchange ${ }^{10}$.

There are limited studies on direct and head-to-head comparison of VectorFlow and Palindrome catheters. The observed findings suggest that more studies with randomization design and longer follow-ups are required.

\section{CONCLUSION}

The VectorFlow hemodialysis catheter has a better survival rate after 6 months, when compared to the Palindrome catheter. We recommend using this catheter in CKD patients who require long-term permanent catheters. Specific design of the tip of the VectorFlow catheter may play an important role in its function and complications rates, influencing adequate hemodialysis treatment of patients. The results obtained from this study provide a general overview to surgeons to consider physical factors such as catheter tip shape.

\section{COMPETING INTERESTS}

The authors declare that they have no conflict of interest.

\section{AUTHORS' CONTRIBUTIONS}

MRS proposed the concept of the research. AV performed data gathering, follow up of the patients, and statistical analyses. MRS \& AV drafted the article and supervised the research.

\section{ETHICAL APPROVAL}

All procedures performed in studies involving human participants were in accordance with the ethical standards of the institutional and/or national research committee and with the 1964 Helsinki declaration and its later amendments or comparable ethical standards.

\section{INFORMED CONSENT}

Informed consent has been obtained from the patient for publication of the case report and accompanying images.

\section{FUNDING}

The authors declare that this study was funded by Kermanshah University of Medical Sciences, Kermanshah, Iran

\section{ACKNOWLEDGMENTS}

This article is the result of the thesis by Ali Veisi (\# 97603), approved by the Research Deputy of Kermanshah University of Medical Sciences. The authors wish to thank all staffs of the Surgery Department of Imam Reza Hospital for their cooperation in this study. We appreciate the Clinical Research Development center of Imam Reza Hospital, Kermanshah University of Medical sciences for their wise advice. 


\section{REFERENCES}

1. Dember LM, Beck GJ, Allon M, Delmez JA, Dixon BS, Greenberg $A$, et al. Effect of clopidogrel on early failure of arteriovenous fistulas for hemodialysis: a randomized controlled trial. JAMA. 2008;299(18):2164-71. PMID: 18477783. Available from: 10 1001/jama.299.18.2164.

2. Tordoir J, Canaud B, Haage P, Konner K, Basci A, Fouque D, et al. EBPG on Vascular Access. Nephrol Dial Transplant. 2007;22:ii88-117. PMID: 17507428. Available from: 10.1093/ ndt/gfm021.

3. Knuttinen MG, Bobra S, Hardman J, Gaba RC, Bui JT, Owens CA A review of evolving dialysis catheter technologies. Semin Intervent Radiol. 2009;26(2):106-14. PMID: 21326500. Available from: 10.1055/s-0029-1222453.

4. Li M, Zhang Z, Yu Y, Chen H, Li X, Ma J, et al. Clinical application of long-term Palindrome catheter in hemodialysis patients. Iran J Kidney Dis. 2014;8(2):123-9. PMID: 24685735.

5. Miller LM, MacRae JM, Kiaii M, Clark E, Dipchand C, Kappel J et al. Hemodialysis Tunneled Catheter Noninfectious Complications. Can J Kidney Health Dis. 2016;3:2054358116669130. PMID: 28270922.

6. Group VAW. Clinical practice guidelines for vascular access. Am J Kidney Dis. 2006;48:176-247. PMID: 16813989. Available from: 10.1053/j.ajkd.2006.04.029.

7. Meersch HVD, Bacquer DD, Vandecasteele SJ, den Bergh BV, Vermeiren $\mathrm{P}$, Letter JD, et al. Hemodialysis catheter design and catheter performance: a randomized controlled trial. Am J Kidney Dis. 2014;64(6):902-8. PMID: 24713222. Available from: 10.1053/j.ajkd.2014.02.017.

8. Tal MG. Comparison of recirculation percentage of the palindrome catheter and standard hemodialysis catheters in a swine model. J Vasc Interv Radiol. 2005;16(9):1237-40. PMID: 16151065. Available from: 10.1097/01.RVI.0000171700.45582. $9 \mathrm{E}$.

9. Mojibian H, Spector M, Ni N, Eliseo D, Pollak J, Tal M. Initial clinical experience with a new heparin-coated chronic hemodialysis catheter. Hemodial Int. 2009;13(3):329-34. PMID: 19473278. Available from: 10.1111/j.1542-4758.2009.00339.x.

10. Clark TW, Redmond JW, Mantell MP, Nadolski GJ, Mondschein Jl, Dowd MF, et al. Initial Clinical Experience: Symmetric-Tip Dialysis Catheter with Helical Flow Characteristics Improves Patient Outcomes. J Vasc Interv Radiol. 2015;26(10):1501-8. PMID: 26239895. Available from: 10.1016/j.jvir.2015.06.033.

11. Ross JR, Puga TA, Philbeck TE. Longitudinal dialysis adequacy and clinical performance of the VectorFlow hemodialysis catheter: a prospective study. . J Vasc Access. 2017;18(6):492497.

12. Clark TW, Isu G, Gallo D, Verdonck P, Morbiducci U. Comparison of symmetric hemodialysis catheters using computational fluid dynamics. J Vasc Interv Radiol. 2015;26(2). PMID: 25645414. Available from: 10.1016/j.jvir.2014.11.004.

13. Wang K, Sun W, Shi X. Upper extremity deep vein thrombosis after migration of peripherally inserted central catheter (PICC): A case report. Medicine (Baltimore). 2017;96(51):e9222. PMID: 29390472. Available from: 10.1097/ MD.0000000000009222. 